\title{
Pneumonia Caused by Emerging Viral Agents
}

\author{
Cecilia Perret Pérez and Marcela Ferrés Garrido
}

\section{Contents}

Middle East Respiratory Syndrome ………………………………………… 335

Severe Acute Respiratory Syndrome/Asian Pneumonia ....................................... 337

HCoV-NL 63 and HCoV-HKU1 .................................................................. 337

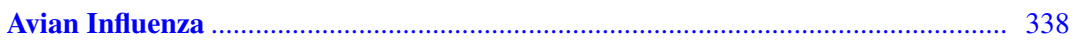

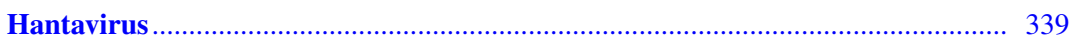

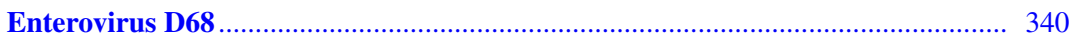

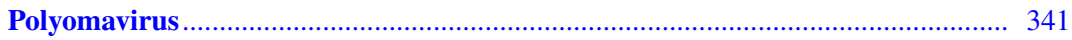

Sources

Emerging viruses that cause pneumonia in humans have a common trait: they are all zoonoses. Normally, these agents circulate in the animal population, often without causing morbidity, but under certain circumstances they can move to human hosts, which determines the occurrence of a new type of disease. Nature holds many examples of such diseases, including avian influenza, Ebola, and severe acute respiratory syndrome

C. Perret Pérez · M. Ferrés Garrido $(\square)$ Department of Pediatrics, School of Medicine, Pontificia Universidad Católica de Chile, Santiago, Chile

e-mail: cperret@med.puc.cl; mferres@med.puc.cl
(SARS). In this chapter we review agents that have provoked major concern outside their country of origin, but also Hanta virus, because of the endemic nature it has acquired in Chile.

\section{Middle East Respiratory Syndrome}

The Middle East respiratory syndrome (MERS) is caused by MERS-CoV, a recently identified coronavirus. Several coronaviruses can cause upper respiratory tract infections, but in some cases they can also produce lower respiratory tract infections and flu-like states. The SARS coronavirus and MERS-CoV are two pathogens from the coronavirus family that predominantly cause serious lower tract respiratory infections with a high mortality rate, but they are genetically different viruses. 
MERS-CoV was first identified in 2012 following the death of a patient in Saudi Arabia with a serious respiratory infection. This finding led to the retrospective diagnosis of the first cases of an inhospital outbreak during 2012 in Jordan. Most cases have been recorded in the Middle East, with more than $75 \%$ of the cases in Saudi Arabia and some cases outside this region affecting travelers. The mortality rate of MERS is approximately $30 \%$.

Information about the MERS mechanism of transmission is still limited, but it is likely to occur through droplets and by direct and indirect contact with infected respiratory secretions. Aerosol transmission has not been ruled out. Currently, transmission between humans is limited and occasional, with a low secondary attack rate. Isolated cases have been recorded, consisting of nosocomial and household outbreaks, but transmission is not sustained over time. This was the situation in the major South Korean outbreak, which originated through a case in Saudi Arabia and affected more than 180 people. Adequate infection control measures in healthcare rapidly limit in-hospital transmission.
Genetic analysis shows that the human MERS$\mathrm{CoV}$ is quite similar to the virus found in bats and essentially identical to that observed in camels. The virus appears to have originated in bats, transitioning through camels, probably in Africa, and afterward being transmitted to humans. Serological studies do not show its presence in humans before 2012, but it has been observed in camels since the 1990s. This observation suggests that camels are the reservoirs of the virus, which can be transmitted to humans through direct contact with these animals or through consumption of their milk: 1599 cases had been diagnosed by July 2015, with 574 deaths [World Health Organization (WHO)]. Of these patients, 63\% are male, with an average age of 48 years (Fig. 34.1). In children, the disease tends to be milder or asymptomatic, with severe cases resulting from a comorbidity. Incubation, defined as the period between the primary and the secondary case, is estimated to last an average of 5 days (2-14 days).

Clinical presentation is characterized by fever, cough, myalgia, and diarrhea. The disease has a wide symptomatic spectrum, which can range

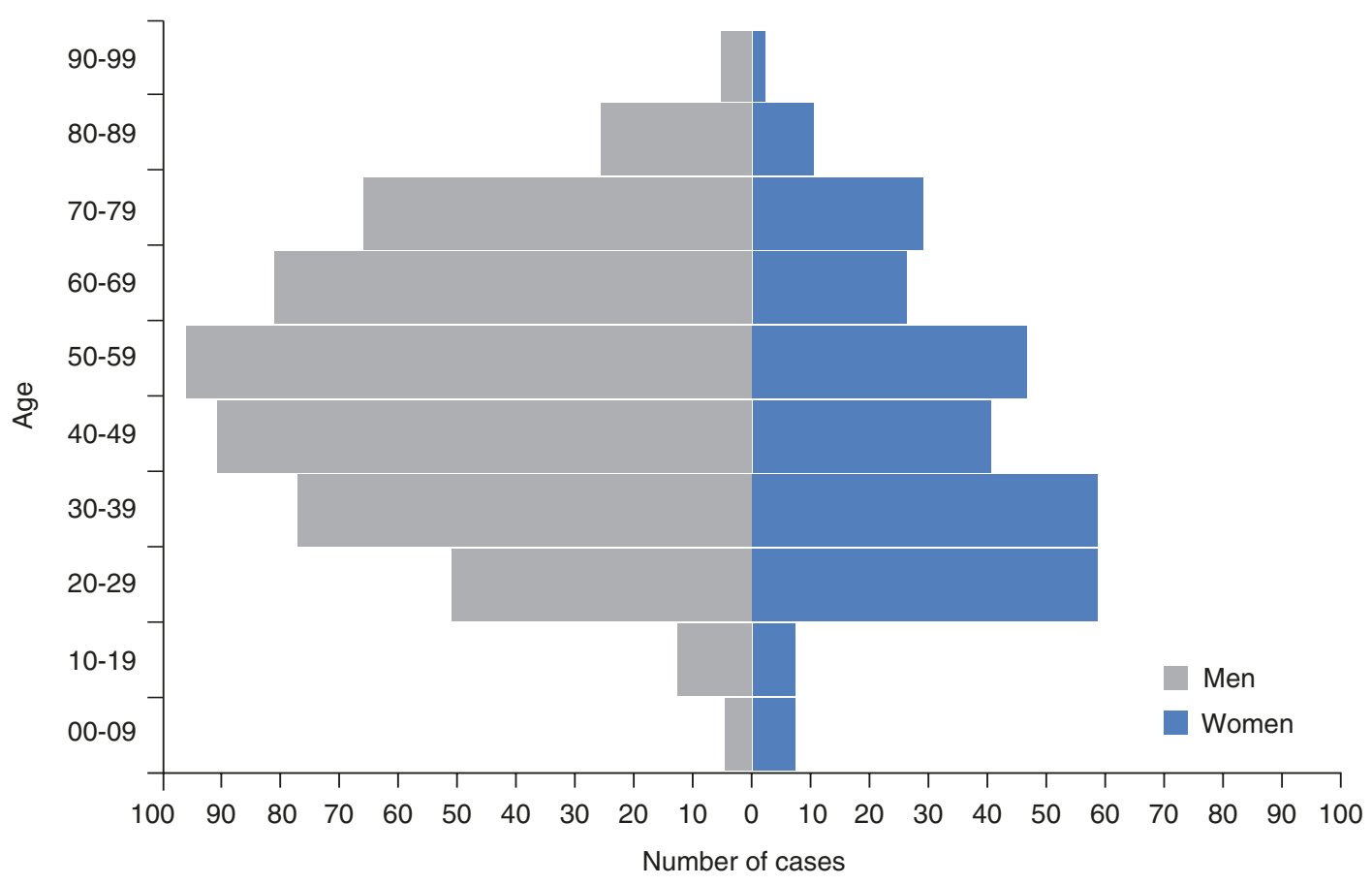

Fig. 34.1 Age distribution for MERS. (Source: http://ecdc.europa.eu/en/publications/Publications/RRA-MERS-CoVthirteenth-update.pdf) 
from asymptomatic infections to fulminant respiratory failure, which is related to high mortality. Patients evolve rapidly (5-day period on average) to respiratory and kidney failure, requiring ventilation support and intensive care management in more than $60 \%$ of cases. Diagnostic confirmation is achieved through viral isolation in laboratories with biosecurity clearance level 3; alternatively, the virus is detected in respiratory samples through molecular biology techniques (polymerase chain reaction, PCR), which are only available in reference laboratories. In patients who have displayed symptoms for more than 14 days, the determination of specific antibodies is recommended.

There is no specific treatment, because no antiviral therapy is available, only support measures in intensive care for the most serious cases. There is no specific preventive vaccine. This infection should be suspected in every traveler who reports having traveled to the Arabian Peninsula during the past 14 days and who also presents with respiratory symptoms and fever.

\section{Severe Acute Respiratory Syndrome/Asian Pneumonia}

SARS is the first identified coronavirus capable of producing a serious pulmonary disease, unlike those previously known $(\mathrm{HCoV}-229 \mathrm{E}$ and HCoV-OC 43), which cause the common cold. SARS emerged in late 2002 in China and quickly disseminated through Southeast Asia, Europe, Africa, and America. Bats were its presumed reservoir, and it was transferred to humans through civets. This virus caused great international alarm because of its rapid progression and its seriousness, resulting from extensive and rapidly progressing pneumonia with a mortality rate around $50 \%$. Health workers were particularly affected. The most constant symptoms during admissions were high fever, malaise, cough, and headache, followed by diarrhea and prolonged fever.

More than 8000 cases were noted, causing more than 700 deaths. Thanks to international coordination under WHO leadership, the epidemic was controlled in July 2003. There were five clear objectives, which were achieved in record time: identification of the etiological agent, development of diagnostic tests for virus detection, creation and evaluation of epidemiological treatment protocols to reduce morbidity and mortality, definition of key epidemiological parameters to control transmission, and formulation of appropriate public health measures.

SARS demonstrated that coronaviruses can cause serious lower respiratory infections, which would later be observed for HCoV-NL63 and HCoV-HKU 1. To date, no new circulation of this agent has been proven.

\section{HCoV-NL 63 and HCoV-HKU1}

Coronavirus NL 63, which belongs to coronavirus group I, was discovered for the first time in a child with bronchiolitis in the Netherlands in 2004, and then ratified in small children hospitalized for serious respiratory infections. HKU1, which belongs to the coronavirus group II identified in 2005, was also shown to be capable of causing a lower respiratory tract infection. Both have been described throughout the world, proving their ubiquity. This virus is detected in up to $10 \%$ of acute respiratory disease cases, and its symptoms range from upper respiratory disease, including flu-like disease, fever, rhinitis, odynophagia, and cough, to serious conditions with a rapidly progressing respiratory disease. Infection by $\mathrm{HCoV}-\mathrm{N} 63$ in children manifests as an obstructive laryngotracheitis in up to $45 \%$ of cases in comparison with children not infected by this virus.

The first HCoV-HKU1 viruses were described in elderly patients with preexisting conditions that caused their death. Some later studies, which considered children as well as adults, have confirmed that infection caused by this coronavirus may worsen the health status of individuals with underlying diseases, so that they need to be hospitalized more often. As with HcoV-N63, infection causes upper respiratory tract symptoms, such as fever, coryza, odynophagia, and coughing. Wheezing, pneumonia, and bronchiolitis may also be present. In children a greater fre- 
quency of feverish convulsions has been observed in comparison with children who were not infected by HCoV-HKU1.

HCoV-NL63 and HCoV-HKU1 are viruses that tend to manifest as a common cold, just as the usual coronaviruses $\mathrm{HCoV}-229 \mathrm{E}$ and HCoV-OC43; nevertheless, in small children, elderly patients, and immunosuppressed patients, they can cause serious respiratory disease with a high mortality rate.

\section{Avian Influenza}

The influenza A virus is widespread in nature: its main reservoir is domestic and feral birds. Several types exist, according to their hemagglutinin $(\mathrm{H})$ and neuraminidase $(\mathrm{N})$ makeup, only two of which have recently circulated among humans and are causing seasonal outbreaks: H1N1 and H3N2.

The animal reservoir of the virus is large and varied. Several subtypes have been described, which include 17 hemagglutinin and 9 neuraminidase types. These zoonotic viruses may cause diseases and infections in several animals, generally pigs and poultry. These zoonotic viruses in particular adapt very poorly to humans, so they seldom cause human diseases, which are limited to outbreaks circumscribed in terms of time and population. Nevertheless, human cases of A H5N1 were observed in Hong Kong for the first time in 1997 and have continued being reported to date. More than 700 cases have been notified, all in Asia and Northern Africa, with 413 deaths. Most cases are isolated, with some intrafamilial clusters being described. Human-to-human transmission is rare, which reflects the poor ability of the virus to adapt to human respiratory mucosa. The persistence of the circulation of these viruses and the great genetic variability of the influenza A virus make us fear that the avian virus may adapt to humans, which would ease its transmission among the human population, potentially causing a pandemic: this is what happened with the H1N1 influenza A, which had a porcine origin and adapted to humans, creating a pandemic in 2009.
The first cases of human infection caused by H7N9 influenza A were reported in China during 2013, and most of these were related in one way or another to contact with poultry, whether direct or environmental in markets where live birds are commercialized. By 2015 there had been 488 confirmed H7N9 influenza cases in China since the beginning of the outbreak, with 185 deaths. This virus does not seem to spread easily among humans, and there has been no proof of sustained human transmission, although its transmission potential seems to be more effective than that of the H5N1 influenza A virus. A couple of cases have been reported outside China: a traveler in Malaysia who stayed in China and a man and his wife who were diagnosed in Canada in January 2015 after traveling to China (WHO). There are no recorded secondary cases in these countries, which confirms its low probability of human-tohuman transmission.

Cases have been observed during the coldest seasons in China. From December 2014 to February 2015, 83 new cases were diagnosed, with an average age of 56 years and 19 deaths. Of these newly diagnosed patients, $72 \%$ are male, and $93 \%$ of patients have had direct contact with poultry markets. The incubation period is 4 days (2-8 days). Even though mild cases have been described, most diagnoses have been serious, with a mortality rate close to $35 \%$. Hospitalized patients have a febrile disease, with temperatures above $102.2^{\circ} \mathrm{F}$ and cough. The disease progresses swiftly from moderate to severe. In contrast to human seasonal influenza, most patients do not report rhinorrhea or odynophagia.

In a set of hospitalized patients, the disease progressed swiftly for an average of 3 days after its onset, counting from the beginning of the symptoms until hospitalization. Almost $70 \%$ required invasive mechanical ventilation, with a death rate of $30 \%$. Deaths from respiratory failure reached $38 \%$, and $62 \%$ were caused by septic shock. Most deaths corresponded to elderly patients and with a underlying disease, as well as to the use of systemic steroids. Diagnosis is performed by identifying viral RNA through RT-PCR in respiratory tract samples, obtained through swabbing or nasal suctioning. 
As well as other avian influenza A virus, this virus is sensitive to oseltamivir and is resistant to adamantines. Oseltamivir is indicated for hospitalized or ambulatory patients, whether they have been confirmed or are under suspicion of being infected by H7N9 influenza, even if more than $48 \mathrm{~h}$ have passed since the onset of symptoms. The dosage and timeframe of therapy are not clearly established for serious patients, but a longer timeframe is suggested, around 10 days and in higher doses. In patients with mild and noncomplicated infections, therapy must continue for 5 days. Patients with mild infection, who require ambulatory treatment and whose only exposure factor is travel to an area where there are recorded cases, in humans or birds, have no empirical indication for oseltamivir.

Isolated cases of avian origin in humans caused by the influenza H10N8 virus and H6N1 have been observed in China. These facts prove that in these zoonotic influenza cases vigilance is extremely important, given its pandemic potential, so it is crucial to pay close attention to travelers and enforce local vigilance.

\section{Hantavirus}

In 1993, a new virus from the the Bunyaviridae family was identified in the United States of America. It was named "virus with no name" and was deemed responsible for what is now known as cardiopulmonary hantavirus syndrome (síndrome cardiopulmonar por hantavirus, SCPH), a feverish disease characterized by respiratory insufficiency and shock. This discovery, which was a new zoonosis, in practice extended over the next years across the whole American continent. In this process, new clinical manifestations were recognized and new agents identified, including the Andes, Laguna Negra, Araquara, and Choclo viruses, which are prevalent in Chile, Argentina, Paraguay, Brazil, and Panama, respectively.

Their natural reservoir are Sigmondontinae rodents, which belong to the Muridae family. These animals develop a chronic infection with an intermittent viral and asymptomatic excretion. Stress situations, such as lack of food during birthing periods, cold, or habitat interventions such as logging, have been associated with greater virus excretion in these rodents. Mice excrete the virus through their feces, urine, and saliva, contaminating the environment. The most representative virus-carrying rodents are Peromyscus maniculatus or "deer mouse" (which carries the no-name virus), Oligoryzomys longicaudatus or "long-tailed mouse," and Calomys laucha, among others. Humans acquire the infection through the inhalation of secretions (stools, urine, saliva) of infected rodents. The Andes virus, predominant in Southern Argentina and the single causal agent in Chile, is only transmitted through close human-to-human contact.

Hantaviruses are spherical viruses with a trisegmented RNA genome with a lipid envelope through which two glycoproteins ( $\mathrm{Gn}$ and $\mathrm{Gc}$ ) protrude. These three segments code through proteins such as RNA polymerase (L segment); glycoproteins $\mathrm{Gn}$ and $\mathrm{Gc}$ (M segment), which are important in the recognition of $\beta 3$ integrins that the virus use as receptors; and nucleoprotein ( $\mathrm{S}$ segment), a highly conserved protein used for the laboratory diagnosis of these agents. The lipid envelope is sensitive and is destroyed by detergents, chloride, desiccation, and sun exposure. All these actions form the basis of the prevention and control recommendations for hantavirus infections.

The agent enters the respiratory tract through inhalation of the aerosolized virus in the environment or through contaminated human secretions. After an incubation period that ranges from 1 to 6 weeks (18 days on average), nonspecific symptoms begin, including fever, myalgias, and headache, plus digestive symptoms (more common in children). This stage is followed by progressive respiratory disease and finally by respiratory failure, which is the most serious manifestation of this infection. In $100 \%$ of the cases of acute infection, the virus is present in all the white cells and, in variable proportions, in plasma, respiratory secretions, saliva, and urine. Also, viral RNA has been detected in white cells up to 15 days before and 90 days after the first symptoms.

Interaction with $\beta 3$ integrins and the replication of viral endothelial cells of various tissues 
appear to alter the modulation functions of permeability in these cells, especially increasing the permeability in small lung vessels, which favors arterial hypotension, thrombocytopenia, and hypoxia from plasma flooding into alveolar spaces. Protein $\mathrm{N}$ and superficial glycoproteins stimulate the production of specific and neutralizing antibodies, which have been associated with better survival outcomes when they increase prematurely.

Patients who progress to the cardiopulmonary phase, where respiratory failure sets in, require supplementary oxygen; in addition, more than two thirds need mechanical ventilation, and $50 \%$ of patients develop cardiogenic shock, which constitutes a poor prognosis factor. The increased vascularity rate explains the pulmonary edema observed during this stage. This functional alteration is transitory, lasting from 48 to $72 \mathrm{~h}$; afterward, pulmonary function is quickly recovered following a brief period of noticeable diuresis. Cardiogenic shock is difficult to manage and is the main cause of death. The lethality of hanta cardiopulmonary syndrome is about $35 \%$, and most deaths occur during the first $48 \mathrm{~h}$ of evolution.

The hemogram is the most useful general laboratory test for hypothesizing a diagnosis, because it can, from an early stage, reveal manifest reductions in the number of platelets as well as the presence of lymphocytes, which take the shape of immunoblasts. A late onset of hematocrit increase has been observed, which is concomitant with the beginning of the cardiopulmonary phase of the disease.

It is also helpful to test for LDH and transaminases, which increase nonspecifically. A chest $\mathrm{X}$-ray may change in a matter of hours from a nonspecific interstitial pattern to diffuse pulmonary edema. The virological diagnosis is confirmed through RT-PCR in white cells or through the ELISA detection of specific IgM/IgG for each regional virus.

Currently, there is no specific treatment for hantavirus infection other than cardiopulmonary support. Patient should be monitored closely, preferably in an intensive care unit, to provide measures such as mechanical ventilation for breathing support, restriction of liquids, and vasoactive drugs. The use of an antiviral such as ribavirin has not been shown to be an effective treatment. Extracorporeal membrane oxygenation (ECMO) has been used as a rescue therapy in some cases of serious cardiopulmonary failure that do not respond to conventional ventilation and vasoactive drugs.

In Chile, two therapeutic options have been investigated for hantavirus: methylprednisolone in high doses and immune plasma with high neutralizing antibody titers. Only the latter strategy has shown promising results relative to mortality rate reduction when used immediately after symptom onset.

As a control measure in hospitalized patient management, and considering that hantavirus has been described as capable of causing nosocomial transmission, standard precautions must be taken: ideally, interning the patient in an individual room and wearing protection equipment [apron, gloves, face mask (no. 95) with a high efficiency filter, and security glasses], especially in procedures during which there is close contact with the patient's fluids, such as intubation, secretion suctioning, and retrieval of samples for laboratory tests.

\section{Enterovirus D68}

This viral agent, enterovirus D68, has been known since 1962, when it was isolated in children suffering from bronchiolitis and pneumonia. Because no widely available trials have been conducted, not much is known about its epidemiology and clinical manifestations. During the fall of 2014, Missouri and Illinois hospitals reported an unusual rise in the number of serious cases of children, with or without a background of obstructive disease, who presented with acute respiratory infection. Enterovirus D65 was detected in these patients by applying molecular biology techniques to respiratory samples.

The infection is more frequent in school-age children, whose most relevant clinical antecedent was the presence of previous persistent coughing, 
asthma, or wheezing episodes that may have required intensive care unit (ICU) management. The virulence of this agent is more impressive than that of other enteroviruses, considering that it was also identified as a causal agent of obstructive episodes in previously healthy children who, when treated with antiasthma therapy, did not respond adequately and had to be hospitalized in intensive care because of hypoxemia and, with some exceptions, had to receive mechanical ventilation.

The most common laboratory findings were high total neutrophils and chest X-ray showing peribronchial interstitial infiltrations, hyperinsufflation, and atelectasis.

As diagnostic tests improve in sensitivity and specificity, and their use becomes more widespread in pediatric centers, our understanding of the epidemiology and pathogeny of this viral agent will increase.

\section{Polyomavirus}

Two polyomaviruses with respiratory tropism were discovered in 2007 through deep sequencing of samples taken from respiratory secretions of symptomatic patients: polyomaviruses KI (KIPyV) and WU (WUPyV). These viruses can be found in the lower and upper respiratory tract of immunocompetent as well as immunocompromised patients. Their pathogenic role is not completely clear, because they usually occur at low frequency, have a low viral load, and are related to pathogens whose morbidity is better characterized. Diagnosis requires molecular techniques (real-time PCR), whose inclusion in future research will allow us to better characterize the epidemiology and clinical spectrum of the infections caused by these agents.

\section{Sources}

Arabi YM, Arifi AA, Balkhy HH, Najm Hani, Aldawood AS, Ghabashi A et al. Clinical course and outcomes of critically ill patients with Middle East respiratory syndrome coronavirus infection. Ann Intern Med 2014;160:389-397.

Confirmed human cases of avian influenza A (H7N9) reported to WHO. Report 18: data in WHO/HQ as of 14 July 2001. Disponible en: http://www.who.int/influenza/human_animal_interface/influenza_h7n9/18_ report-webh7n9number_20140714.pdf?ua=1.

Ferres M, Vial P, Marco C, Yanez L, Godoy P, Castillo C, et al. Prospective evaluation of household contacts of persons with hantavirus cardiopulmonary syndrome in Chile. J Infect Dis. 2007;195(11):1563-71.

Figueiredo LT, Souza WM, Ferres M, Enria DA. Hantaviruses and cardiopulmonary syndrome in South America. Virus Res. 2014;187:43-54.

Forster J, Ihorst G, Rieger CH, Stephan V, Frank HD, Gurthet $\mathrm{H}$, et al. Prospective population-based study of viral lower respiratory tract infections in children under 3 years of age (the PRIDE study). Eur J Pediatr. 2014;163:709-16.

Gao R, Cao B, Hu Y, Feng Z, Wang D, Hu W, et al. Human infection with a novel Avian-origin influenza A (H7N9) virus. N Engl J Med. 2013;368:1888-97.

Manigold T, Vial P. Human hantavirus infections: epidemiology, clinical features, pathogenesis and immunology. Swiss Med Wkly. 2014;144:w13937.

Martinez-Valdebenito C, Calvo M, Vial C, Mansilla R, Marco C, Palma RE, et al. Person-to-person household and nosocomial transmission of Andes hantavirus, Southern Chile, 2011. Emerg Infect Dis. 2014;20(10):1629-36.

Memish ZA, Al-Tawfiq JA, Assiri A, Al Rabiah FA, Al Hajjar S, Albarrak A, et al. Middle East respiratory syndrome coronavirus disease in children. Pediatr Infect Dis J. 2014;33:904-6.

MINSAL. Guía Clínica de Prevención, Diagnóstico y Trata-miento del Sindrome Cardiopulmonar por Hantavirus web site MINSAL; 2013.

Pyrc K, Berkhout B, van der Hoek L. The novel human coronaviruses NL63 and HKU1. J Virol. 2007;81(7):3051-7.

Rha B, Rudd J, Feikin D, Watson J, Curns AT, Swerdlow DL, et al. Update on the epidemiology of Middle East Respiratory Syndrome Coronavirus (MERS-CoV) infection, and guidance for the public, clinicians, and public health authorities: January 2015. MMWR. 2015;64(3):61-2.

Schountz T, Prescott J. Hantavirus immunology of rodent reservoirs: current status and future directions. Viruses. 2014;6(3):1317-35. https://doi.org/10.3390/ v6031317.

WHO. Middle East respiratory syndrome coronavirus (MERS-CoV). Summary of Current Situation, Literature Update and Risk Assessment. 7 July 2015 WHO/MERS/RA/15.1.

Yang Y, Guo F, Zhao W, Gu Q, Huang M, Cao Q, et al. Novel Avian-Origin Influenza A (H7N9) in critically ill patients in China. Crit Care Med. 2015;43:339-45. 